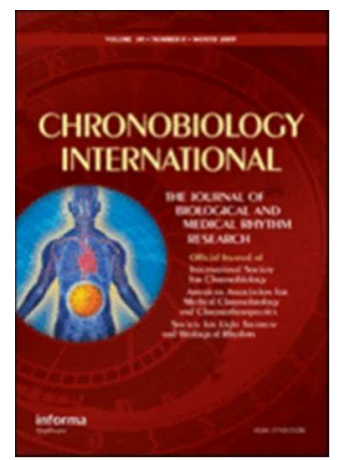

\title{
Exploring the effect of chronotype on hippocampal volume and shape: A combined approach.
}

\begin{tabular}{|r|l|}
\hline Journal: & Chronobiology International \\
\hline Manuscript ID & LCBI-2018-0055 \\
\hline Manuscript Type: & Short Communications \\
\hline Date Submitted by the Author: & 07-Mar-2018 \\
\hline Complete List of Authors: & $\begin{array}{l}\text { Horne, Charlotte; University of Roehampton, Psychology } \\
\text { Norbury, Ray; University of Roehampton, Psychology }\end{array}$ \\
\hline Keywords: & $\begin{array}{l}\text { Chronotype, Hippocampus, Emotion, Depression, Emotion regulation, } \\
\text { Morphology }\end{array}$ \\
\hline
\end{tabular}

SCHOLARONE

Manuscripts 
Exploring the effect of chronotype on hippocampal volume

and shape: A combined approach.

Charlotte Mary Horne ${ }^{1}$ and Ray Norbury ${ }^{1,2}$

${ }^{1}$ Department of Psychology

University of Roehampton

${ }^{2}$ Address for correspondence:

Dr Ray Norbury

Department of Psychology

University of Roehampton

Whitelands College,

London

SW15 4JD

E: ray.norbury@roehampton.ac.uk

T: +44(0) 2089325788 


\begin{abstract}
Current evidence suggests that acute depression is associated with reduced total hippocampal volume and regional atrophy. Here, using structural magnetic resonance imaging, we assayed linear effects of chronotype on total hippocampal volume and morphology. Later chronotype was associated with localised atrophy in the subiculum region of the right hippocampus in the absence of changes in total volume. The hippocampus forms a key node in a network of brain regions implicated in emotional regulation and alterations in the structure of this region may underpin, in part, the increased vulnerability for depression in late chronotype individuals.
\end{abstract}

Keywords: Chronotype, Hippocampus, Emotion, Depression, Regulation, Morphology

Total word count: 4180 (including table and figure legends)

Abstract word count: 94

2 


\section{Introduction}

Major Depressive Disorder (MDD) is one of the most prevalent and debilitating of all psychiatric disorders, affecting 4-17\% of the general population (Wong \& Licinio, 2001). Fewer than half of patients reach remission with standard pharmacotherapy and nearly $80 \%$ of patients suffer relapse. Often starting in adolescence (Kessler, Avenevoli, \& Ries Merikangas, 2001), MDD follows a chronic course with a high risk of recurrence or persistence into adulthood and is associated with ongoing psychosocial difficulties and elevated risk for general medical illness (Nicholson, Kuper, \& Hemingway, 2006). This malignant pattern of psychosocial dysfunction and disability suggests that the most effective strategies to reduce the health burden of MDD would be obtained through the targeting of 'atrisk' individuals.

A number of risk factors for depression have been identified including positive family history (Wells, Deykin, \& Klerman, 1985), female gender (Piccinelli \& Wilkinson, 2000), neuroticism (Vittengl, 2017) and childhood abuse (Widom, DuMont, \& Czaja, 2007). Increasing evidence also identifies late chronotype as a vulnerability factor for depression (Fabbian et al., 2016). Across the general population late chronotype is associated with increased depressive symptomatology (Levandovski et al., 2011; Merikanto et al., 2013, 2015; Togo, Yoshizaki, \& Komatsu, 2017), diagnosis of depression and use of antidepressant medication (Merikanto et al., 2013, 2015). Together, these data demonstrate a clear association between late chronotype and depression but the observational nature of these studies prevents examination of the underlying mechanisms that confer risk.

In the context of depression, one structure that has received significant critical inspection is the hippocampus (Rogers, Renoir, \& Hannan, 2017). For example, the hippocampus is densely innervated by 5-HT (serotonin) projections from the dorsal raphe. Serotoninergic signalling is strongly implicated in a number of psychiatric disorders 3 
including depression and plays a key role in the cognitive and emotional processing that underlies them. The hippocampus is highly connected to other subcortical regions involved in emotional processing (e.g. amygdala) and also the hypothalamus (which forms part of the stress-related hypothalamic-adrenal-pituitary axis (HPA axis)). Moreover, it has been demonstrated that functionally relevant levels of neurogenesis occur in the adult human hippocampus (Spalding et al., 2013) and adult hippocampal neurogenesis is implicated in antidepressant response, stress response and cognitive function (O'Leary \& Cryan, 2014).

Due to the implicated role of the hippocampus in medication response, stress and cognition, a large number of Magnetic Resonance Imaging (MRI) studies have investigated hippocampal volume in MDD. Although equivocal (Bora, Fornito, Pantelis, \& Yücel, 2012), a number of meta-analyses indicate reductions in hippocampal volume in patients $v s$. controls (Arnone, McIntosh, Ebmeier, Munafò, \& Anderson, 2012; MacQueen et al., 2003; Schmaal et al., 2015). It is unclear, however, if these reductions are an outcome of the onset of clinical symptoms (i.e. the neurotoxicity hypothesis (Sheline, 2011)) or predate the onset of the disorder (i.e. the vulnerability hypothesis (Baaré et al., 2010; Durmusoglu et al., 2018)). Support for the vulnerability hypothesis can be found across a number of studies that have demonstrated reduced hippocampal volume and/or shape differences in euthymic participants with positive family history for depression (Baaré et al., 2010; Chen, Hamilton, \& Gotlib, 2010; Durmusoglu et al., 2018) and early-life adversity (Dahmen et al., 2017) two well recognised risk factors for depression. To our best knowledge, however, no one has yet investigated hippocampal volume or shape and related this to chronotype. Based on the extant literature and consistent with the vulnerability hypothesis, we predicted that later chronotype would be associated with lower total hippocampal (volumetric analysis) and localised atrophy (vertex analysis). 
Methods

\section{Participants}

The study was approved by the local ethics committee and written informed consent was obtained prior to any study procedures taking place. A total of 50 participants were recruited, all were in good physical health and free of concurrent medication. Exclusion criteria were current or previous depression, presence of major depression in a biological parent, diagnosed sleep disorder (each assessed by self-report) and contraindication for MR examination. Chronotype, sleep quality and depression/anxiety were determined using, respectively, the 5-item reduced Morningness-Eveningness Questionnaire (Adan \& Almirall, 1991) the Pittsburgh Sleep Quality Index (Buysse, Reynolds, Monk, Berman, \& Kupfer, 1989). and the Patient Health Questionniare-4 (Löwe et al., 2010). Please see Table 1 for details.

\section{TABLE 1 NEAR HERE PLEASE}

\section{Image data acquisition}

All imaging data were acquired on a research dedicated 3T Magnetom TIM Trio (Siemens, Erlangen, Germany) fitted with a 32-channel head coil and located at the Combined Universities Brain Imaging Centre (CUBIC). For each participant, we collected a $\mathrm{T}_{1}$-weighted whole-brain scan (magnetization-prepared rapid acquisition with gradient echo (MPRAGE), inversion time $(\mathrm{TI})=1100 \mathrm{~ms}$, repetition time $(\mathrm{TR})=1830 \mathrm{~ms}$, echo time $(\mathrm{TE})=3.03 \mathrm{~ms}$, flip angle $(\mathrm{FA})=11^{\circ}$, field of view $(\mathrm{FOV})=256 \times 256 \times 160 \mathrm{~mm}^{3}$, voxel size $\left.=1 \times 1 \times 1 \mathrm{~mm}^{3}\right)$. 


\section{Data analysis pipeline}

All image pre-processing and analyses were performed using FSL version 5.0.10 (FMRIB Software Library, http://fsl.fmrib.ox.ac.uk/fsl/fslwiki/). Non-brain removal was performed on each individual's $\mathrm{T}_{1}$-weighted image using a deformable model that evolves to fit the brain's surface by the application of a set of locally adaptive model forces. Subcortical brain measures (bilateral hippocampus) were delineated using FMRIB's Integration Registration and Segmentation Tool (FIRST). FIRST is a model-based segmentation tool that utilises shape and appearance models taken from an atlas of manually segmented images from the Centre for Morphometric Analysis. These manual segmentations are parameterised as surface meshes from which a point distribution is modelled. Using the observed intensities from each individual's $T_{1}$-weighted image FIRST finds the most probable shape by searching through linear combinations of shape variation modes resulting in a hippocampus segmentation for each individual in native space. At each step, processed images were carefully inspected by one of the authors $(\mathrm{CH})$ to ensure accuracy of results. Intracranial volume (ICV) was estimated as the inverse of the determinant of the rotation-translation matrix obtained after affine registration to a standard template multiplied by the template volume and used to correct for differences in head size.

In addition to volumetric analyses, vertex analyses were also conducted to investigate localised shape differences in the hippocampus. Based on the segmentation described above the boundary for each structure (left and right hippocampus) were reconstructed into a vertexbased surface and the vertex locations for each subject projected on the surfaces of the average shape transformed to Montreal Neurological Institute space. The projections were scalar values representing the signed, perpendicular distance from the average surface, where negative/positive values indicate, respectively, greater inversion/eversion from the average hippocampal shape. Linear effects of chronotype on scalar projection values were tested for 6 
significance using non-parametric permutation tests (applying 5000 permutations) with sleep quality, age, gender and mood/anxiety included as covariates in the regression model. Control of the family-wise error rate was obtained using cluster-based thresholding with an initial threshold of $\mathrm{Z}>3.1$ and a corrected spatial extent threshold of $p=.05$.

\title{
Results
}

Right and left hippocampal volumes as percentage of ICV $(M=.00265$, SD .0004; $M$ $=.00262, \mathrm{SD} .0004)$ were similar to previously published findings (Dean et al., 2016). There were no laterality effects (dependent samples $t(49)=.693, p=.49$ ) or association with rMEQ $($ right: $r(50)=.085, p=.56$; left $r(50)=.060, p=.69)$. Vertex analyses showed a significant relationship between vertex height and chronotype such that increased inversion (atrophy) was associated with later chronotype in a mid-anterior region of the right hippocampus (please see Figure 1). There was no relationship between localised shape change and chronotype in left hippocampus.

\author{
FIGURE 1 NEAR HERE PLEASE
}




\section{Discussion}

The principle aims of the current work were to investigate hippocampal volume and shape in never-depressed healthy participants and relate this to chronotype. Consistent with our original hypotheses later chronotype was associated with localised atrophy in right hippocampus independent of age, gender sleep quality and mood. However, contrary to our predictions, there was no relationship between total hippocampal volume and chronotype.

Interpretation of the current findings should take into consideration a number of limitations. Current, previous and family history of depression was determined using selfreport. Future studies may benefit from reference to medical history or structured clinical interview to assess exclusion criteria. We also did not measure or exclude participants with high neuroticism which is another population proven to be at-risk of depression (Kendler, Gatz, Gardner, \& Pedersen, 2006). However, previous work suggests that, in the age range included in this study, neuroticism does not impact on hippocampal volume (Chan et al., 2016). Of note, repeated clinical observations have reported an association between acute depression and increased availability of cortisol. The hippocampus is enriched with glucocorticoid receptors and elevated cortisol secretion is associated with reduced neurogenesis and neurotoxicity (Cowen, 2010). We did not measure cortisol levels in our participants so cannot exclude a potential impact of this hormone on our findings. Arguing against this however, a number of studies have reported increased cortisol waking response and higher overall concentrations of cortisol in early vs. late chronotypes (Kudielka, Federenko, Hellhammer, \& Wüst, 2006; Maierova et al., 2016). In respect to risk for depression, an elevated waking cortisol response has been reported in some groups at risk for depression (e.g. positive family history, Mannie, Harmer, \& Cowen, 2007) but not others (e.g high neuroticism, Chan, Harmer, Goodwin, \& Norbury, 2008). As we excluded participants with a positive family history of depression and current, although limited, evidence suggests 8 
early chronotype is associated with higher levels of cortisol we would argue that fluctuation in cortisol levels is an unlikely explanation for our findings.

Current evidence indicates the acute depression is associated with modest reductions in total hippocampal volume (Arnone et al., 2012; MacQueen et al., 2003; Schmaal et al., 2015). The temporal characteristics of these changes, however, are not fully understood. The neurotoxicity hypothesis posits that volumetric changes are a neurodegenerative response to chronic stress (Sheline, 2011). The biological abnormalities underlying this volume reduction are likely multifactorial, including prolonged exposure to glucocorticoids and stress-induced reductions in neurotrophic factors and neurogenesis. In support of the neurotoxicity hypothesis a number of studies have reported that cumulative duration of lifetime depression, number of depressive episodes and an earlier age of depression onset are associated with hippocampal volume loss (MacQueen et al., 2003; Sheline, Sanghavi, Mintun, \& Gado, 1999).

Hippocampal volume reduction has also been reported in groups at increased risk for depression. For example, Chen and colleagues (2010) observed reduced hippocampal volume in adolescent daughters of mothers with recurrent episodes of depression that occurred during the daughter's lifetime (at-risk group) as compared with age-matched daughters of mothers with no history of depression. Similarly, Amico et al., (2011) compared hippocampal volume in currently depressed, at-risk (family history of depression) and control adults (Amico et al., 2011). The high-risk group had smaller right hippocampal volume than the depressed and low-risk group (high-risk $<$ depressed $<$ low-risk) a finding that may be consistent with plasticity effects of antidepressant treatment (O’Leary \& Cryan, 2014). Of note, Chan and colleagues (Chan et al., 2016) compared hippocampal volume in older adult highly neurotic individuals (another established risk factor depression), recovered depressed patients and agematched low neuroticism controls. They reported larger bilateral hippocampal volumes in the 9 
at-risk group as compared to the recovered depressed and low neuroticism groups. The authors concluded that the larger hippocampal volume in this older adult (mean age 40 years) at-risk group may reflect a resilience factor. In a complimentary study the same authors compared high and low neurotic younger adults (mean age 20 years) and found no differences in hippocampal volume (Chan et al., 2016).

We did not observe an association between later chronotypes and global hippocampal volume. We did, however, observe more subtle localised atrophy related to chronotype in a mid-anterior region of the right hippocampus. Indeed, a number of studies in depressed patients have revealed localised shape changes in the absence of global reductions in volume. For example, Isikli et al., (2013) reported bilateral hippocampal shape alterations in currently depressed patients as compared to a matched group of healthy controls in a number of regions including an area on the inferior surface of the right hippocampus (subiculum) similar to that reported here. Similarly, Posener and colleagues, using high dimensional mapping of the hippocampus, reported inward deformations in the right subiculum of currently depressed patients (Posener et al., 2003). Bearden et al., (2009) observed that more severe symptom severity in unmedicated currently depressed patients was associated with greater left hippocampal atrophy in Cornu Ammonis (CA) 1 and subiculum (Bearden et al., 2009). More recently, Watanabe and colleagues (Watanabe et al., 2017) reported significant inward deformations in bilateral CA 1 and subiculum in first episode drug-naïve depressed patients as compared to a demographically similar helahty control group. In addition, within the patient group there was a linear relationship between the degree of deformation and serum cortisol levels.

The above findings suggest that localised hippocampal atrophy, particularly in CA1 and subiculum, is present during the acutely depressed state. Data on whether these morphological changes are present prior to the onset of disease (vulnerability factor) are more 10 
limited. In support of the vulnerability hypothesis Durmusoglu and colleagues reported contractions in the right $\mathrm{CA} 1$ /subiculum border in young adult women at increased risk for depression by virtue of a mother with a diagnosis of recurrent depression (Durmusoglu et al., 2018). These data are similar to our own findings and provide further evidence that in some vulnerable individuals localised hippocampal atrophy precedes the onset of depression and, therefore, may be implicated in the aetiology of the disorder.

The trajectory and functional significance of the localised atrophy reported here are unknown. It is possible that the extent and degree of atrophy increases over time with the accumulation of atrophy ultimately leading to clinical depression. In addition, the hippocampus is critically involved with emotion regulation (Amico et al., 2011) and projects to a number of limbic regions including the amygdala. Here we did not measure emotion regulation directly but previous work suggests that later chronotype is associated with maladaptive emotion regulation strategies (reduced cognitive reappraisal and increased emotion suppression, Watts \& Norbury, 2017) and greater rumination and cognitive reactivity (Antypa et al., 2017). Moreover, late chronotype has been associated with reduced microstructural integrity in the white matter underlying the left anterior cingulate cortex (ACC; Rosenberg, Maximov, Reske, Grinberg, \& Shah, 2014) and an elevated amygdala response to negative emotional stimuli and reduced amygdala-ACC functional connectivity (Horne \& Norbury, 2018). Together, these regions form key nodes in a network of brain areas implicated in emotional regulation (Hiser \& Koenigs, 2017) and alterations in the structure and function of these regions may underpin, in part, the increased vulnerability for depression in late chronotype individuals. Future, well-powered, prospective studies are required to investigate and better understand the importance of the structural and functional changes reported here and elsewhere (Rosenberg et al., 2014) for the development of depression in late chronotype individuals. 


\section{References}

Adan, A., \& Almirall, H. (1991). Horne \& Östberg morningness-eveningness questionnaire: A reduced scale. Personality and Individual Differences, 12(3), 241-253. https://doi.org/10.1016/0191-8869(91)90110-W

Amico, F., Meisenzahl, E., Koutsouleris, N., Reiser, M., Möller, H.-J., \& Frodl, T. (2011). Structural MRI correlates for vulnerability and resilience to major depressive disorder. Journal of Psychiatry \& Neuroscience: JPN, 36(1), 15-22. https://doi.org/10.1503/jpn.090186

Antypa, N., Verkuil, B., Molendijk, M., Schoevers, R., Penninx, B. W. J. H., \& Van Der Does, W. (2017). Associations between chronotypes and psychological vulnerability factors of depression. Chronobiology International, 1-11. https://doi.org/10.1080/07420528.2017.1345932

Arnone, D., McIntosh, A. M., Ebmeier, K. P., Munafò, M. R., \& Anderson, I. M. (2012). Magnetic resonance imaging studies in unipolar depression: Systematic review and meta-regression analyses. European Neuropsychopharmacology, 22(1), 1-16. https://doi.org/10.1016/j.euroneuro.2011.05.003

Baaré, W. F. C., Vinberg, M., Knudsen, G. M., Paulson, O. B., Langkilde, A. R., Jernigan, T. L., \& Kessing, L. V. (2010). Hippocampal volume changes in healthy subjects at risk of unipolar depression. Journal of Psychiatric Research, 44(10), 655-662. https://doi.org/10.1016/j.jpsychires.2009.12.009

Bearden, C. E., Thompson, P. M., Avedissian, C., Klunder, A. D., Nicoletti, M., Dierschke, N., ... Soares, J. C. (2009). Altered hippocampal morphology in unmedicated patients with major depressive illness. ASN NEURO, 1(4). https://doi.org/10.1042/AN20090026

Bora, E., Fornito, A., Pantelis, C., \& Yücel, M. (2012). Gray matter abnormalities in Major Depressive Disorder: a meta-analysis of voxel based morphometry studies. Journal of Affective Disorders, 138(1-2), 9-18. https://doi.org/10.1016/j.jad.2011.03.049 
Buysse, D. J., Reynolds, C. F., 3rd, Monk, T. H., Berman, S. R., \& Kupfer, D. J. (1989). The Pittsburgh Sleep Quality Index: a new instrument for psychiatric practice and research. Psychiatry Research, 28(2), 193-213.

Chan, S., Harmer, C. J., Goodwin, G. M., \& Norbury, R. (2008). Risk for depression is associated with neural biases in emotional categorisation. Neuropsychologia, 46(12), 2896-2903. https://doi.org/10.1016/j.neuropsychologia.2008.05.030

Chan, S., Harmer, C. J., Norbury, R., O’Sullivan, U., Goodwin, G. M., \& Portella, M. J. (2016). Hippocampal volume in vulnerability and resilience to depression. Journal of Affective Disorders, 189, 199-202. https://doi.org/10.1016/j.jad.2015.09.021

Chen, M. C., Hamilton, J. P., \& Gotlib, I. H. (2010). Decreased Hippocampal Volume in Healthy Girls at Risk of Depression. Archives of General Psychiatry, 67(3), 270. https://doi.org/10.1001/archgenpsychiatry.2009.202

Cowen, P. J. (2010). Not fade away: the HPA axis and depression. Psychological Medicine, 40(1), 14. https://doi.org/10.1017/S0033291709005558

Dahmen, B., Puetz, V. B., Scharke, W., Polier, G. G. von, Herpertz-Dahlmann, B., \& Konrad, K. (2017). Effects of Early-Life Adversity on Hippocampal Structures and Associated HPA Axis Functions. Developmental Neuroscience, (0). https://doi.org/10.1159/000484238

Dean, D. J., Orr, J. M., Bernard, J. A., Gupta, T., Pelletier-Baldelli, A., Carol, E. E., \& Mittal, V. A. (2016). Hippocampal Shape Abnormalities Predict Symptom Progression in Neuroleptic-Free Youth at Ultrahigh Risk for Psychosis. Schizophrenia Bulletin, 42(1), 161-169. https://doi.org/10.1093/schbul/sbv086

Durmusoglu, E., Ugurlu, O., Akan, S., Simsek, F., Kizilates, G., Kitis, O., ... Gonul, A. S. (2018). Hippocampal shape alterations in healthy young women with familial risk for unipolar depression. Comprehensive Psychiatry, 82, 7-13. https://doi.org/10.1016/j.comppsych.2018.01.004

Fabbian, F., Zucchi, B., Giorgi, A. D., Tiseo, R., Boari, B., Salmi, R., ... Manfredini, R. (2016). Chronotype, gender and general health. Chronobiology International, 1-20. https://doi.org/10.1080/07420528.2016.1176927 
Hiser, J., \& Koenigs, M. (2017). The Multifaceted Role of the Ventromedial Prefrontal Cortex in Emotion, Decision Making, Social Cognition, and Psychopathology. Biological Psychiatry. https://doi.org/10.1016/j.biopsych.2017.10.030

Horne, C. M., \& Norbury, R. (2018). Late chronotype is associated with enhanced amygdala reactivity and reduced fronto-limbic functional connectivity to fearful versus happy facial expressions. NeuroImage, 171, 355-363. https://doi.org/10.1016/j.neuroimage.2018.01.025

Kendler, K. S., Gatz, M., Gardner, C. O., \& Pedersen, N. L. (2006). Personality and major depression: a Swedish longitudinal, population-based twin study. Archives of General Psychiatry, 63(10), 1113-1120. https://doi.org/10.1001/archpsyc.63.10.1113

Kessler, R. C., Avenevoli, S., \& Ries Merikangas, K. (2001). Mood disorders in children and adolescents: an epidemiologic perspective. Biological Psychiatry, 49(12), 1002-1014.

Kudielka, B. M., Federenko, I. S., Hellhammer, D. H., \& Wüst, S. (2006). Morningness and eveningness: the free cortisol rise after awakening in 'early birds' and 'night owls'. Biological Psychology, 72(2), 141-146. https://doi.org/10.1016/j.biopsycho.2005.08.003

Levandovski, R., Dantas, G., Fernandes, L. C., Caumo, W., Torres, I., Roenneberg, T., ... Allebrandt, K. V. (2011). Depression Scores Associate With Chronotype and Social Jetlag in a Rural Population. Chronobiology International, 28(9), 771-778. https://doi.org/10.3109/07420528.2011.602445

Löwe, B., Wahl, I., Rose, M., Spitzer, C., Glaesmer, H., Wingenfeld, K., ... Brähler, E. (2010). A 4item measure of depression and anxiety: validation and standardization of the Patient Health Questionnaire-4 (PHQ-4) in the general population. Journal of Affective Disorders, 122(1-2), 86-95. https://doi.org/10.1016/j.jad.2009.06.019

MacQueen, G. M., Campbell, S., McEwen, B. S., Macdonald, K., Amano, S., Joffe, R. T., ... Young, L. T. (2003). Course of illness, hippocampal function, and hippocampal volume in major depression. Proceedings of the National Academy of Sciences, 100(3), 1387-1392. https://doi.org/10.1073/pnas.0337481100

14 
Maierova, L., Borisuit, A., Scartezzini, J.-L., Jaeggi, S. M., Schmidt, C., \& Münch, M. (2016). Diurnal variations of hormonal secretion, alertness and cognition in extreme chronotypes under different lighting conditions. Scientific Reports, 6. https://doi.org/10.1038/srep33591

Mannie, Z. N., Harmer, C. J., \& Cowen, P. J. (2007). Increased waking salivary cortisol levels in young people at familial risk of depression. The American Journal of Psychiatry, 164(4), 617-621. https://doi.org/10.1176/appi.ajp.164.4.617

Merikanto, I., Kronholm, E., Peltonen, M., Laatikainen, T., Vartiainen, E., \& Partonen, T. (2015). Circadian preference links to depression in general adult population. Journal of Affective Disorders, 188, 143-148. https://doi.org/10.1016/j.jad.2015.08.061

Merikanto, I., Lahti, T., Kronholm, E., Peltonen, M., Laatikainen, T., Vartiainen, E., ... Partonen, T. (2013). Evening types are prone to depression. Chronobiology International, 30(5), 719-725. https://doi.org/10.3109/07420528.2013.784770

Nicholson, A., Kuper, H., \& Hemingway, H. (2006). Depression as an aetiologic and prognostic factor in coronary heart disease: a meta-analysis of 6362 events among 146538 participants in 54 observational studies. European Heart Journal, 27(23), 2763-2774. https://doi.org/10.1093/eurheartj/ehl338

O’Leary, O. F., \& Cryan, J. F. (2014). A ventral view on antidepressant action: roles for adult hippocampal neurogenesis along the dorsoventral axis. Trends in Pharmacological Sciences, 35(12), 675-687. https://doi.org/10.1016/j.tips.2014.09.011

Piccinelli, M., \& Wilkinson, G. (2000). Gender differences in depression: Critical review. The British Journal of Psychiatry, 177(6), 486-492. https://doi.org/10.1192/bjp.177.6.486

Posener, J. A., Wang, L., Price, J. L., Gado, M. H., Province, M. A., Miller, M. I., ... Csernansky, J. G. (2003). High-dimensional mapping of the hippocampus in depression. The American Journal of Psychiatry, 160(1), 83-89. https://doi.org/10.1176/appi.ajp.160.1.83

Rogers, J., Renoir, T., \& Hannan, A. J. (2017). Gene-environment interactions informing therapeutic approaches to cognitive and affective disorders. Neuropharmacology. https://doi.org/10.1016/j.neuropharm.2017.12.038 
Rosenberg, J., Maximov, I. I., Reske, M., Grinberg, F., \& Shah, N. J. (2014). 'Early to bed, early to rise': diffusion tensor imaging identifies chronotype-specificity. NeuroImage, 84, 428-434. https://doi.org/10.1016/j.neuroimage.2013.07.086

Schmaal, L., Veltman, D. J., van Erp, T. G. M., Sämann, P. G., Frodl, T., Jahanshad, N., ... Hibar, D. P. (2015). Subcortical brain alterations in major depressive disorder: findings from the ENIGMA Major Depressive Disorder working group. Molecular Psychiatry. https://doi.org/10.1038/mp.2015.69

Sheline, Y. I. (2011). Depression and the Hippocampus: Cause or Effect? Biological Psychiatry, 70(4), 308-309. https://doi.org/10.1016/j.biopsych.2011.06.006

Sheline, Y. I., Sanghavi, M., Mintun, M. A., \& Gado, M. H. (1999). Depression duration but not age predicts hippocampal volume loss in medically healthy women with recurrent major depression. The Journal of Neuroscience: The Official Journal of the Society for Neuroscience, 19(12), 5034-5043.

Spalding, K. L., Bergmann, O., Alkass, K., Bernard, S., Salehpour, M., Huttner, H. B., ... Frisén, J. (2013). Dynamics of Hippocampal Neurogenesis in Adult Humans. Cell, 153(6), 1219-1227. https://doi.org/10.1016/j.cell.2013.05.002

Togo, F., Yoshizaki, T., \& Komatsu, T. (2017). Association between depressive symptoms and morningness-eveningness, sleep duration and rotating shift work in Japanese nurses. Chronobiology International, 1-11. https://doi.org/10.1080/07420528.2016.1273942

Vittengl, J. R. (2017). Who pays the price for high neuroticism? Moderators of longitudinal risks for depression and anxiety. Psychological Medicine, 1-12. https://doi.org/10.1017/S0033291717000253

Watanabe, R., Kakeda, S., Watanabe, K., Liu, X., Katsuki, A., Umeno-Nakano, W., ... Korogi, Y. (2017). Relationship between the hippocampal shape abnormality and serum cortisol levels in first-episode and drug-naïve major depressive disorder patients. Depression and Anxiety, 34(5), 401-409. https://doi.org/10.1002/da.22604

Watts, A. L., \& Norbury, R. (2017). Reduced Effective Emotion Regulation in Night Owls. Journal of Biological Rhythms, 32(4), 369-375. https://doi.org/10.1177/0748730417709111

16 
Wells, V. E., Deykin, E. Y., \& Klerman, G. L. (1985). Risk factors for depression in adolescence. Psychiatric Developments, 3(1), 83-108.

Widom, C. S., DuMont, K., \& Czaja, S. J. (2007). A prospective investigation of major depressive disorder and comorbidity in abused and neglected children grown up. Archives of General Psychiatry, 64(1), 49-56. https://doi.org/10.1001/archpsyc.64.1.49

Wong, M.-L., \& Licinio, J. (2001). Research and treatment approaches to depression. Nature Reviews Neuroscience, 2(5), 343-351. https://doi.org/10.1038/35072566

\section{Acknowledgements}

We would like to thank Mr Ari Lingeswaran, Royal Holloway University for his technical assistance and all of our volunteers who participated in this work.

\section{Competing interests}

The authors report no conflicts of interest.

\section{Funding}

This work was supported by the University of Roehampton.

17 


\begin{tabular}{cccc}
\hline Variable & $M($ SD) & Range & Range \\
Age & $21.24(3.77)$ & $18-37$ & (this sample) \\
(original scale) \\
Gender & Female 38 (76\%); & - & - \\
Sleep quality & Male 12 (24\%) & $1-15$ & $0-21$ \\
Chronotype & $7.30(3.42)$ & $6-20$ & $4-25$ \\
PHQ-4 (Anxiety) & $12.62(3.62)$ & $0-5$ & $0-6$ \\
PHQ-4 (Mood) & $0.91(1.09)$ & $0-6$ & $0-6$ \\
& $1.61(1.58)$ & & \\
\hline
\end{tabular}

Table 1. Basic demographics, sleep quality (PSQI), chronotype (rMEQ), mood and anxiety (PHQ-4). Please see Methods for questionnaire details. Values show mean (SD). Also included are the questionnaire range (minimum-maximum) for the study sample and original scale. 


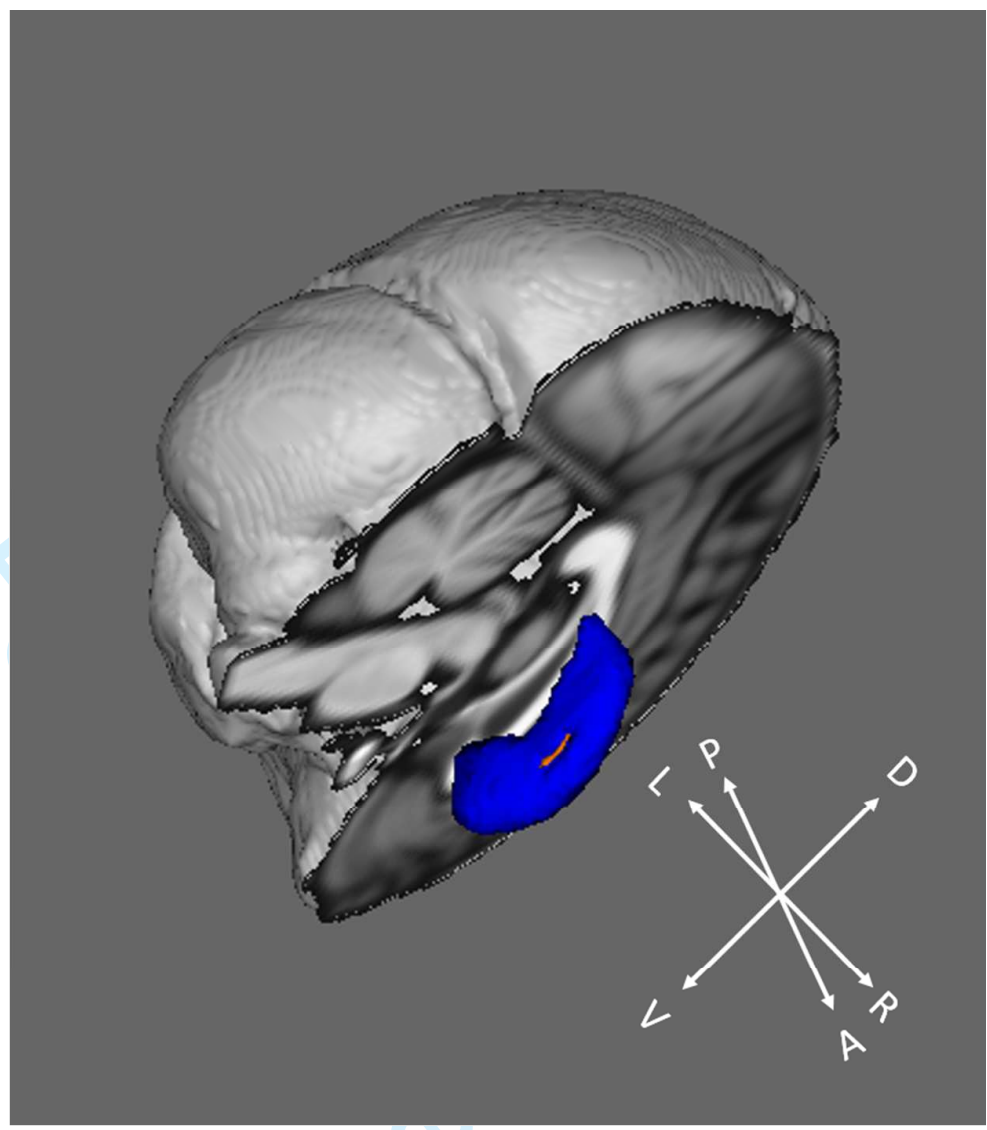

Figure 1. Vertex analysis shows focal atrophy (shown in red) associated with later chronotype on the ventral aspect of the mid-anterior right hippocampus. V,D,L,R,P,A; ventral, dorsal, left, right, posterior, anterior. 